\title{
Anti-cyclic citrullinated peptide antibodies in rheumatoid arthritis, brucellar arthritis and Behcet's disease
}

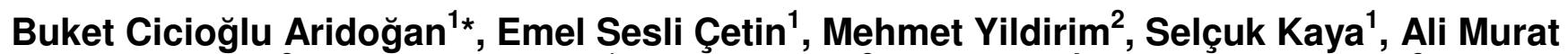 \\ Ceyhan $^{2}$, Mustafa Demirci ${ }^{1}$, Serpil Savaş ${ }^{3}$, Onur Ural ${ }^{4}$ and Selami Akkuş ${ }^{3}$ \\ ${ }^{1}$ Department of Microbiology and Clinical Microbiology, Faculty of Medicine, Suleyman Demirel University, Isparta, \\ Turkey. \\ ${ }^{2}$ Department of Dermatology, Faculty of Medicine, Suleyman Demirel University, Turkey. \\ ${ }^{3}$ Department of Microbiology and Clinical Microbiology, Faculty of Medicine, Katip Çelebi University İzmir, Turkey. \\ ${ }^{4}$ Department of Physical Therapy and Rehabilitation, Faculty of Medicine, Suleyman Demirel University Isparta, Turkey. \\ ${ }^{5}$ Department of Infectious diseases, Faculty of Medicine, Selçuklu, Selcuk University, Turkey. \\ ${ }^{6}$ Department of Physical Therapy and Rehabilitation, Faculty of Medicine, Yıldırım Beyazıt University Ankara, Turkey.
}

Accepted 23 August, 2011

\begin{abstract}
In Behcet's disease (BD) and Brucellosis, joint involvement is very common and it may share similarities with rheumatoid arthritis (RA). Anti-cyclic citrullinated peptides (CCP) are evaluated as more specific autoantibodies than rheumatoid factor (RF) in RA. The aim of this study is to determine whether anti-CCP antibodies are useful in the differential diagnosis of RA from other arthritis or not. 153 serum samples were used in the study; 34 from RA patients, 26 from brucellar arthritis, 20 from BD with arthritis, 41 from BD without arthritis and 32 from healthy controls. In vitro quantitative, anti-CCP levels were measured by using fluoroenzyme-immunoassay (FEIA) method. Among RA patients, $73.5 \%$ were anti-CCP-positive and $70.5 \%$ were RF-positive. While there was a statistical difference in the anti-CCP and RF positivity rates between RA and brucellar arthritis patients, BD patients and healthy controls $(p=$ 0.0001), there was no statistical difference between BD patients with arthritis and BD patients without arthritis. Also none of the Brucellar arthritis patients was positive for anti-CCP antibodies, one of them were positive for RF. Our results indicate that anti-CCP antibodies are not associated with brucellar arthritis or BD with joint involvement and since they show high specificity and good sensitivity for RA, they can be evaluated as a better marker than RF especially for the differentiation of RA from other types of arthritis such as BD and brucellar arthritis.
\end{abstract}

Key words: Rheumatoid arthritis, Behcet's disease, brucellar arthritis, anti-cyclic citrullinated peptide antibody, rheumatoid factor.

\section{INTRODUCTION}

Citrulline is a non-standard amino acid, created by deimination of arginine residues in several proteins by the action of peptidylarginine deiminase. Citrullinated extracellular fibrin in the rheumatoid arthritis (RA) synovium may be one of the major autoantigens affecting the local immune

${ }^{*}$ Corresponding author. E-mail: drbuket@gmail.com. Tel: 00902462113679 . Fax: 00902462371762. response, suggested by the discovery of local production of anti-cyclic citrullinated peptide (CCP) and anti-citrullinated filaggrin antibodies in the joint (Wiik and van Venrooij, 2003). The antibodies to synthetic cyclic citrullinated peptides (anti-CCP) are even more specific for RA, and may also have prognostic capacity (Forslind et al., 2004; Schellekens et al., 2000).

$\mathrm{RA}$ is a chronic, systemic autoimmune disease which causes severe destruction of joints in the hands and feet due to chronic inflammation and is characterized by 
symmetric, erosive joint synovitis (Arıdoğan et al., 2008). But it is often not diagnosed until permanent rheumatic changes have occurred besides having positive test results for rheumatoid factor (RF), and the addition of CCP antibodies to the testing profile is a welcome addition for the diagnosis of patients with RA (Moore, 2006).

Behcet's disease (BD) is an idiopathic, chronic, recurrent and multisystem disease characterized by oral and genital ulcers, ocular inflammation, synovitis, and thrombophlebitis. The disease is characterized by endothelial dysfunction and may produce a wide variety of symptoms (Moore, 2006). BD has a worldwide distribution. The incidence of this disease varies by region. The highest prevalence is reported in Turkey, with 80 to 420 cases per 100,000 of the population, followed by Japan, where there are 13 to70 cases per 100,000 of the population (Yates and Michelson, 2006; Yamamoto et al., 1974; Yurkadul et al., 1988). Joint involvement in BD is very common, occurring in 45 to $55 \%$ of the patients. The most common signs and symptoms include arthralgias, redness, and swelling of the joints. Joint involvement is usually asymmetrical and recurrent (Yates and Michelson, 2006).

Brucellosis is a zoonotic disease with a worldwide distribution, and it is still an important public health problem in Turkey because of the consumption of unpasteurized dairy products (Memisoglu et al., 2008; Aygen et al, 1995). Brucellosis has non-specific clinical manifestations. In addition to being a systemic illness, brucellosis may affect many systems including liver, hematologic system, bones and joints (including arthritis, spondylitis, and osteomyelitis (Shapiro et al., 1998). The musculoskeletal system is one of the most commonly affected systems and the most frequently affected bones are femur, tibia and humerus. Especially in children and young adults, the most frequent monoarthritis involvement is shown on the knee or ankle (Memisoglu et al., 2008; Gotuzzo et al., 1982).

In $\mathrm{BD}$ and brucellosis joint involvement is very common and it may share similarities with RA and differential diagnosis from RA of other arthritis is very important. The aim of this study is to evaluate whether anti-CCP antibodies are useful in differentiating the diagnosis of RA from other arthritis. For this purpose, we compared antiCCP antibodies of patients with BD and brucellosis who have joint involvement, with RA and healthy control subjects.

\section{MATERIALS AND METHODS}

One hundred and fifty three serum samples were used in the study; 34 from RA patients, 26 from brucellar arthritis patients, and 61 from patients with BD (20 with arthritis, 41 without arthritis) and 32 from healthy controls. RA patients included 29 women with the mean age $53.7 \pm 8.6$ (range 40 to 70 ). RA was diagnosed according to the American College of Rheumatology (ACR) criteria (Arnett et al., 1988) and BD was diagnosed for international study group for Behcet's Disease (The international study group for Behcet's disease, 1990). BD patients with arthritis included 14 women whose mean age was $35+7.7$ (range 25 to 49 ). BD patients without arthritis included 20 women with the mean age of $39.7+12.1$ (range 16 to 65). Brucellar arthritis patients included 17 men and 9 women their mean age was $39.8+10.2$ (range 22 to 65 ). All of brucellar arthritis patients were acute brucellosis except two; these patients had relapsed from brucellosis. The sera of patients with brucellar arthritis were obtained from the Department of Infectious Diseases, Medical Faculty of Meram, University of Selcuk, and the sera of patients with RA and patients with BD from the Department of Physical Therapy and Rehabilitation and the Department of Dermatology, Medical Faculty, Suleyman Demirel University. The study was carried out in the Microbiology Laboratory of the Medical Faculty, Suleyman Demirel University. All the patients in the study were informed of the study and they all gave their consent to this study.

In vitro quantitative anti-CCP levels of sera were measured by using fluoroenzyme-immunoassay (FEIA) method (Uni-CAP PharmaciaUpjohn). According to test procedure, the levels between 0.4 to $7 \mathrm{U} / \mathrm{ml}$ were considered as normal values, higher levels from $10 \mathrm{U} / \mathrm{ml}$ were considered as positive and the range was 0.4 to $340 \mathrm{U} / \mathrm{ml}$. The quantitative levels of RF were measured by immunonephelometry (Prospec, Dade Behring) techniques. Titers higher than $15 \mathrm{IU} / \mathrm{ml}$ were considered as positive for RF.

\section{Statistics}

The frequencies were calculated for each group and comparisons for categorical variables were made using the Chi-square test. Continuous variables were compared with Student's t test and Mann Whitney. Scheffe and Tukey-Oneway post-hoc multiple comparisons between all groups were performed on the ranks of the original levels of the parameters. The statistical significance level was set to 0.05 and the SPSS for windows software, version 10.0 (SPSS, Chicago, IL) was used for the analysis.

\section{RESULTS}

Among 34 RA patients, $70.5 \%$ (24) were RF-positive and $73.5 \%$ (25) were anti-CCP-positive. Twenty-two of the 34 RA patients $(64.7 \%)$ had both anti-CCP antibodies and $\mathrm{RF}$, and $20.5 \%$ (7 out of 34 ) of the patients had neither anti-CCP nor RF positivity. While none of the Brucellar arthritis patients was positive for anti-CCP antibodies, one of them was positive for RF. Similarly, while none of the $\mathrm{BD}$ patients with arthritis was positive for anti-CCP antibodies, one of them was positive for RF. In addition, none of the patients with BD without arthritis and control group was positive for anti-CCP antibodies and RF. Thus, the sensitivity and specificity of anti-CCP reactivity for the diagnosis of RA were 73.5 and $100 \%$, respectively; the corresponding results for RF were 70.5 and $98.3 \%$, respectively.

Anti-CCP and RF positivity rates of RA patients were statistically different from Brucellar arthritis, BD patients and healthy controls $(p=0.0001)$. There was no statistical difference between BD patients with arthritis and without arthritis. Also we did not find any statistical difference between the patient groups of BD and Brucellar arthritis with healthy controls.

The mean values of serum anti-CCP antibody and RF in RA patients, Brucella arthritis, BD patients with arthritis, 
Table 1. Demographic and serologic data of the RA, BD (with arthritis and without arthritis), Brucellar arthritis and HC groups.

\begin{tabular}{lccccc}
\hline & RA & Brucellar arthritis & BD with arthritis & BD without arthritis & Healthy control \\
\hline Age, (mean \pm SD) & $53.8 \pm 8.6$ & $39.8 \pm 10.2$ & $35 \pm 7.7$ & $39.7 \pm 12.1$ & $47.5 \pm 6.8$ \\
Female & $29 / 34$ & $9 / 26$ & $14 / 20$ & $20 / 41$ & $24 / 32$ \\
Anti-CCP positivity (\%) & $73.5 \%$ & $0 \%$ & $0 \%$ & $0 \%$ & $0 \%$ \\
Anti-CCP levels & $74.6 \pm 64.9$ & $1.5 \pm 1.4$ & $1.7 \pm 0.7$ & $0.4 \pm 0.1$ & $0.9 \pm 0.6$ \\
RF positivity (\%) & $70.5 \%$ & $3.8 \%$ & $5 \%$ & $0 \%$ & $0 \%$ \\
RF levels & $62.6 \pm 84.8$ & $14 \pm 22.8$ & $10.1 \pm 2.5$ & $9.4 \pm 0.3$ & $9.5 \pm 1$ \\
\hline
\end{tabular}

RA: rheumatoid arthritis; BD: Behcet's disease; RF: rheumatoid factor; anti-CCP: anti-cyclic citrullinated peptide.

and BD patients without arthritis are given on Table 1. Serum levels of anti-CCP and RF were significantly increased in patients with RA in comparison with other groups $(p<0.001$, Oneway Anova, post Hoc Test Scheffe).

In terms of serum anti-CCP antibody levels, we have found statistical difference between RA $(74.6 \pm 64.9)$ and Brucellar arthritis patients $(1.5 \pm 1.4)$ and RA and BD patients with $(1.7 \pm 0.7)$ and without arthritis $(0.4 \pm 0.1)(p$ $=0.0001)$. However, there was no statistical difference between Brucellar arthritis patients and BD patients with arthritis and without arthritis (Table 1). In addition, there was a statistical difference for RF levels between RA patients and Brucellar arthritis patients, RA patients and BD patients with and without arthritis $(p=0.0001)$. We have not found statistical difference between Brucellar arthritis patients and BD patients with controls. There was also no statistical difference between the anti-CCP antibody and RF levels of Brucellar arthritis patients and BD patients with controls.

\section{DISCUSSION}

The differential diagnosis of RA represents both a diagnostic and a therapeutic challenge, since a variety of entities with similar signs and symptoms are prevalent among patients with other types of arthritis (Ceccato et al., 2006). For this purpose, some authors made several investigations for the differential diagnosis of RA from psoriatic arthritis (Inanc et al., 2007). Behcet's disease (Koca et al., 2007), osteoarthritis (Arıdoğan et al., 2008), juvenile idiopathic arthritis (Kasapçopur et al., 2004) etc. However, researchers were unable to find a specific antibody or marker seen in RA. RF has been shown as the most important blood test used to detect RA and to distinguish it from other types of arthritis and other inflammatory processes, but the sensitivity and specificity of RF are not agreeable for all situations. It has been known that many patients with a positive test do not have RA and RF can be negative in patients with RA.

Researchers have reported anti-CCP antibodies as a powerful serologic marker for differential diagnosis of RA especially because of their high sensitivity as well as high specifity (Arıdoğan et al., 2008; Inanc et al., 2007; Koca et al., 2007). Not only did they confirm the diagnosis but also they indicated that the patient is at an increased risk for damage to the joints. However, early tests for CCP showed specificity but lacked sensitivity. With the recent development of third generation enzyme linked immunosorbent assays (ELISA) for CCP, cyclic citrilline peptide antibodies are considered the most sensitive markers for RA (Moore, 2006). Many investigation results concluded that anti-CCP showed higher discriminative ability than other assays, including RF, and would be useful to aid the diagnosis of RA in clinical practice (Suzuki et al., 2003). According to the results of the Foundation for Blood Research, in the sensitivity and specificity of CCP IgG ELISA testing, overall sensitivity of the anti-CCP test was $76 \%$ for RA; $90 \%$ sensitive for RF + RA, and $40 \%$ sensitive for RF- RA. Specificity was $99 \%$ for healthy volunteers, $91 \%$ specific in rheumatic disease controls and $98 \%$ specific in infectious disease controls (The Foundation for Blood Research 2003). In the study of Nishimura and colleagues on the diagnostic accuracy of anti-CCP antibody in RA, it was concluded that although anti-CCP antibodies were more specific than RF, their sensitivity was only $67 \%$ (Nishimura et al., 2007). In addition, false-positive results should be kept in mind with the anti-CCP test because of cross-reactivity to citrullinated proteins found in inflamed joints. Falsepositive results for anti-CCP can be seen in some cases with gout or lupus which has delayed a correct diagnosis. Many authors suggested that the use of both a RF test and an anti-CCP test are better to lead accurate diagnosis of RA (Nishimura et al., 2007; Roubenoff et al., 2008; Arkfeld 2008).

We also performed this study to assess the utility of anti-CCP antibodies in distinguishing patients with RA from patients with BD and brucellosis which have joint involvement. These two diseases, especially Behcet's disease, are among the diseases with high prevalence in Turkey. Also, nearly one third of the brucellosis patients suffer from osteoarticular complications in Turkey (Memisoglu et al., 2008; Hashemi et al., 2007). According to the results of different studies, the frequency of this complication varies from 28 to $70 \%$ depending on the series, the age of the patients, and the infecting Brucella 
species (Hashemi et al., 2007; Taşova et al., 1999; Geyik et al., 2002; Kokoglu et al., 2006). Clinical presentation with negative laboratory test results of brucellosis should not exclude the suspicion of illness (Gotuzzo et al., 1982). In our study we have not determined anti-CCP antibodies in sera of patient with Brucellar arthritis. Although, the relationship between anti-CCP and erosive joint diseases has been shown by some researchers (Schellekens et al., 2000; Inanc et al., 2007; Kasapçopur et al., 2004; Lee and Schur, 2003), we could not find any report about the role of anti-CCP antibodies in the differential diagnosis of RA and brucellar arthritis in the literature. So there is no data in accordance or in contrast with our study.

Behcet's disease is one of the frequent inflammatory diseases in Turkey which may also affect joints (Yates and Michelson, 2006). There are limited data about antiCCP antibody in BD although it has been studied widely in autoimmune diseases with joint involvement, other than RA (Koca et al., 2007; Lee and Schur 2003). In this study, we evaluated anti-CCP antibodies in both serum of BD patients with or without arthritis and the antiCCP antibodies were not determined neither in serum of BD patients with arthritis nor without arthritis. While Sghiri et al. (2007) have reported anti-CCP positivity among BD patients in contrast with our findings; Kogure et al. (2007) have reported that they have not detected any positivity in accordance with the results of our study. Furthermore, only in one study from Turkey we have reached, Koca et al. (2007) have found anti-CCP positivity among $2.2 \%$ of 46 patients with $\mathrm{BD}$, but they have not evaluated $\mathrm{BD}$ patients with arthritis.

In conclusion, our results indicate that anti-CCP antibodies are not associated with Brucellosis or Behcet's diseases which have joint involvement and since they show high specificity and good sensitivity for RA, they can be evaluated as a better marker than RF especially for the differentiation of RA from other types of arthritis such as BD and brucellar arthritis.

\section{REFERENCES}

Arıdoğan BC, Kaya S, Savaş S, Çetin ES, Akkuş S, Demirci M (2008). The role of antı-cyclıc citrullınated peptıde (antı-ccp) antıbodıes in serologic diagnosıs and evaluatıon of disease actıvity in rheumatoıd arthritıs. Mikrobiyol Bul., 42(4): 669-674.

Arkfeld DG (2008). Biological Significance of Anti-Cyclic Citrullinated Peptide Antibody in Rheumatoid Arthritis. Ann. Intern. Med., 148(5): 403.

Arnett FC, Edworthy SM, Bloch DA, McShane DJ, Fries JF, Cooper NS (1988). The American Rheumatism Association 1987 revised criteria for the classification of rheumatoid arthritis. Arthritis Rheum., 31: 315324.

Aygen B, Sumerkan B, Kardeş Y, Doganay M, Inan M (1995). Brucellosis: the evaluation of 183 patients. J. Klimik., 8: 13-16.

Ceccato F, Roverano S, Barrionuevo A, Rillo O, Paira S (2006). The role of anticyclic citrullinated peptide antibodies in the differential diagnosis of elderly-onset rheumatoid arthritis and polymyalgia rheumatica. Clin Rheumatol., 25(6): 854-857

Forslind K, Ahlmen M, Eberhardt K, Hafstrom I, Svensson B (2004). Prediction of radiological outcome in early rheumatoid arthritis in clinical practice: role of antibodies to citrullinated peptides (anti-CCP). Ann Rheum Dis., 63:1090-95.

Geyik MF, Gur A, Nas K, Cevik R, Sarac J, Dikici B (2002). Musculoskeletal involvement of brucellosis in different age groups: a study of 195 cases. Swiss Med Wkly., 132: 98-105.

Gotuzzo E, Alarcon GS, Bocanegra TS, Carrillo C, Guerra JC, Rolando I (1982). Arthricular involvement in human brucellosis: a prospective analysis of 304 cases. Semin Arthritis Rheum., 12:245-255.

Hashemi SH, Keramat F, Ranjbar M, Mamani M, Farzam A, Omidi SJ (2007). Osteoarticular complications of brucellosis in Hamedan, an endemic area in the west of Iran Int. J. Infect. Dis., 11(6): 496-500.

Inanc N, Dalkılıc E, Kamalı S, Kasapoglu-Günal E, Elbir Y, Direskeneli $\mathrm{H}$ (2007). Anti-CCP antibodies in rheumatoid arthritis and psoriatic arthritis. Clin. Rheumatol., 26: 17-23.

Kasapçopur Ö, Altun S, Aslan M, Karaarslan S, Kamburoglu-Göksel A, Sarba S (2004). Diagnostic accuracy of anti-cyclic citrullinated peptide antibodies in juvenile idiopathic arthritis. Ann. Rheum. Dis., 63: 1687-89.

Koca SS, Akbulut H, Dag S, Artas H, Isık A (2007). Anti-Cyclic Citrullinated Peptide Antibodies in Rheumatoid Arthritis and Behcet's Disease. Tohoku J. Exp. Med., 213: 297-304.

Kogure T, Tatsumi T, Fujinaga H, Niizawa A, Terasawa K (2007). Insights to clinical use of serial determination in titers of cyclic citrullinated peptide autoantibodies. Mediators Inflamm., 2007: 12367.

Kokoglu OF, Hosoglu S, Geyik MF, Ayaz C, Akalin S, Buyukbese MA (2006). Clinical and laboratory features of brucellosis in two university hospitals in Southeast Turkey. Trop. Doct., 36: 49-51.

Lee DM, Schur PH (2003). Clinical utility of the anti-CCP assay in patients with rheumatic diseases. Ann. Rheum. Dis., 62: 870-874.

Memisoglu K, Yumuk Z, Akansel G (2008). An unusual presentation of brucellar septic arthritis involving the knee joint with extraarticular hardware: A case report .The Knee., 15(2): 148-150.

Moore E (2006). CCP Antibodies in RA., http://autoimmunedisease.suite101.com/blog.cfm/1549.

Nishimura K, Sugiyama D, Kogata Y, Tsuji G, Nakazawa T, Kawano S (2007). Meta-analysis: diagnostic accuracy of anti-cyclic citrullinated peptide antibody and rheumatoid factor for rheumatoid arthritis. Ann. Intern. Med., 146: 797-808.

Roubenoff R, Weinblatt M, Gregersen Peter K, Arkfeld DG (2008). Biological Significance of Anti-Cyclic Citrullinated Peptide Antibody in Rheumatoid Arthritis. Ann. Intern. Med., 148(5): 403 editor letters

Schellekens GA, Visser $\mathrm{H}$, de Jong BAW, van den Hoogen FHJ, Hazes JMW, Breedveld FC (2000). The diagnostic properties of rheumatoid arthritis antibodies recognizing a cyclic citrullinated peptide. Arthritis Rheum., 43: 155-63.

Sghiri R, Bouagina E, Zaglaoui $H$, Mestiri $H$, Harzallah L, Harrabi I (2007). Diagnostic performances of anti-cyclic citrullinated peptide antibodies in rheumatoid arthritis. Rheumatol Int., 27(12): 1125-30.

Shapiro DS, Wong JD (1998). Brucella. In Manual of Clinical Microbiology, 7th ed., edited by Murray PR, Baron EJ, Pfaller MA, Tenover FC, Yolken RH. ASM Press Washington, D.C. pp. 625-631.

Suzuki K, Sawada T, Murakami A, Matsui T, Tohma S, Nakazono K (2003). High diagnostic performance of ELISA detection of antibodies to citrullinated antigens in rheumatoid arthritis. Scand J. Rheumatol., 32(4): 197-204.

Taşova Y, Saltoğlu N, Şahin G, Aksu HSZ (1999). Osteoarthricular involvement of Brucellosis in Turkey. Clin. Rheumatol., 18: 214-219.

The Foundation for Blood Research. Cyclic Citrullinated Peptide (CCP): A new serum marker for Rheumatoid Arthritis. http://www.fbr.org/home.html Published in January 2003, Updated September 4, 2007

The international study group for Behcet's disease (1990). Criteria for diagnosis of Behcet's disease. Lancet, 335

Wiik AS, van Venrooij WJ (2003). The Use of Anti-cyclic Citrullinated Peptide (anti-CCP) Antibodies in RA. 2003 http://www.rheumatology.org/publications/hotline/1003anticcp.asp.

Yamamoto S, Toyokawa H, Matsubara J (1974). Anation-wide survey of Behcet's disease in Japan: Epidemiological survey. Jpn. J. Ophthalmol., 18: 282-90.

Yates PA, Michelson JB (2006). Behcet Disease. Int Ophthalmol Clin. Spring; 46(2):209-33. 
Yurkadul S, Gunaydin I, Tuzun Y, Tankurt N, Pazarli H, Ozyazgan Y (1988). The prevalence of Behcet's syndrome in a rural area in Northern Turkey. J. Rheumatol., 15: 820-822. 